\title{
Diagnóstico e tratamento da disbiose: Revisão Sistemática
}

\author{
Diagnosis and Treatment of Dysbiosis: Systematic Review
}

Diagnóstico y Tratamiento de la Disbiosis: Revisión Sistemática

Caroline Lobato Pantoja ${ }^{1 *}$, Ana Carolina Cunha Costa ${ }^{1}$, Paula Lavigne de Sousa Costa ${ }^{1}$, Monique de Almeida Hingel Andrade ${ }^{1}$, Victor Vieira Silva ${ }^{1}$, Ana Paula Santos Oliveira Brito ${ }^{1}$, Hamilton Cezar Rocha Garcia'.

\section{RESUMO}

Objetivo: Revisar a literatura a fim de reunir informações atualizadas quanto ao diagnóstico e às condutas terapêuticas disponíveis na disbiose intestinal. Métodos: Foram utilizadas informações obtidas a partir de buscas feitas nas bases de dados BIREME, LILACS, MEDLINE e PUBMED, utilizando como descritores os termos "Disbiose", "Transplante de microbiota fecal" e "Síndrome disabsortiva", com as opções em inglês, português, espanhol e francês, tendo sido empregado o boleano AND. Além disso, foram excluídos do trabalho os artigos publicados há mais de 6 anos, tendo sido encontrados 20 artigos aptos para serem abordados. Vale ressaltar que 18 artigos foram publicados nos últimos 5 anos e apenas 2 artigo foi publicado nos últimos 6 anos. Resultados: Foram coletados 20 artigos em que 9 autores discutiram sobre o tratamento com probióticos, 5 discutiram sobre o tratamento por meio do transplante da microbiota fecal, 5 autores discutiram sobre o tratamento da disbiose com prebióticos, 3 abordaram sobre o tratamento com antibióticos e 3 explicaram sobre o tratamento por meio da suplementação de glutamina. Conclusões: São necessárias maiores pesquisas e estudos sobre o tratamento utilizado na disbiose intestinal para que se tenha uma padronização da técnica.

Palavras-chave: Disbiose, Transplante de microbiota fecal, Probióticos.

\begin{abstract}
Objective: To review the literature and to gather up-to-date information on the diagnosis and therapeutic therapies available in intestinal dysbiosis. Methods: Were use information from searches made in BIREME, LILACS, MEDLINE and PUBMED databases, using the terms "Dysbiosis," "Fecal Microbiotic Transplantation," and "probiotics" as the descriptors. English, Spanish, and Spanish, have been employed the Boolean AND. In addition, writing work for more than 6 years, used for 20 articles, was excluded. It is noteworthy that 18 were launched in the last 5 years and only 2 article was published in the last 6 years. Results: Twenty articles were discussed in which 9 authors discussed probiotic treatment, 5 discussed treatment by fecal microbiota transplantation, 5 authors discussed the treatment of dysbiosis with prebiotics, 3 addressed treatment with antibiotics and 3 explained about treatment by means of glutamine supplementation. Conclusions: These are the largest and most recent studies on the treatment of intestinal disease in order to standardize the technique.
\end{abstract}

Key words: Dysbiosis, Fecal microbiota transplantation, Probiotics.

\section{RESUMEN}

Objetivo: Revisar la literatura para reunir información actualizada sobre el diagnóstico y las conductas terapéuticas disponibles en la disbiosis intestinal. Método: Información se utilizó obtiene a partir de las búsquedas realizadas en las bases de datos de BIREME, LILACS, MEDLINE y PubMed, utilizando como descriptores los términos "disbiosis", "El trasplante de la microbiota fecal" y "probiótico", con opciones en Inglés, Portugués, español y francés, habiendo sido empleado el boleano AND. Además, fueron excluidos del trabajo los artículos publicados hace más de 6 años, habiéndose encontrado 20 artículos aptos para ser abordados. Es importante resaltar que 18 artículos han sido publicados en los últimos 5 años y sólo 2 artículos hasen sido publicado en los últimos 6 años. Resultados: Se recolectaron 20 artículos en los que los 9 autores discutieron sobre el tratamiento con probióticos, 5 discutieron sobre el tratamiento por medio del transplante

${ }^{1}$ Centro Universitário Metropolitano da Amazônia (UNIFAMAZ), Belém-Pará.

*E-mail: pantojacaroline@gmail.com

SUBMETIDO EM: $8 / 2019$ 
de la microbiota fecal, 5 autores discutieron sobre el tratamiento de la disbiosis con prebióticos, 3 abordaron el tratamiento con antibióticos y 3 explicaron sobre el tratamiento por medio de la suplementación de glutamina. Conclusión: Son necesarias mayores investigaciones y estudios sobre el tratamiento utilizado en la disbiosis intestinal para que se tenga una estandarización de la técnica.

Palabras clave: Disbiosis, Trasplante de microbiota fecal, probiótico.

\section{INTRODUÇÃO}

O intestino humano saudável é colonizado por 30 a 400 trilhões de microorganismos e a sua formação inclui bactérias, fungos e vírus. Ao nascer, o trato digestivo humano é estéril, sendo colonizado por determinantes pré-natais, como o modo de parto (principalmente o normal, por ter contato direto com a microbiota fecal da mãe), a idade gestacional, a dieta, o uso de antibióticos, a idade e os microorganismos do trato digestivo materno. Dessa forma, a microbiota intestinal atinge a sua composição adulta cerca dos 3 anos de idade, permanecendo estável por anos, até que alterações no sistema imunológico, fatores genéticos do hospedeiro e fatores ambientais - alimentos, higiene e medicamentos - possam, ocasionalmente, desequilibrar a sua composição (DE OLIVEIRA GLV, et al., 2017; HO KJ e VARGAS J, 2017; PEREIRA IG e FERRAZ IAR, 2017).

A disbiose consiste em um estado mal-adaptativo do microbioma por haver desequilíbrio entre o número de bactérias protetoras e agressoras, tornando o trato gastrointestinal mais vulnerável (PEREIRA IG e FERRAZ IAR, 2017; SIDHU M e VAN DER POORTEN D, 2017). Esse fato pode provocar aumento na permeabilidade intestinal, resultando na passagem ascendente de lipopolissacarídeo (LPS) para a circulação sistêmica, gerando uma endotoxemia metabólica e desenvolvimento de um estado inflamatório crônico (DOS SANTOS MORAES M, et al., 2018).

Essa instabilidade, principalmente em pacientes de UTI, tem sido associada ao aumento da incidência de sepse e morte, uma vez que estudos mostraram que $60-70 \%$ dos pacientes da UTI recebem antibioticoterapia, o que impulsiona a disbiose intestinal. Além disso, muitas doenças gastrointestinais têm sido associadas com alterações no microbioma intestinal, assim como distúrbios metabólicos, doenças hepáticas, distúrbios neurológicos e do humor, artrite, condições imunológicas, a obesidade, doenças cardiovasculares e o câncer colorretal (BRUNEAU A, et al., 2018; MORROW LE e WISCHMEYER P, 2017; SIDHU M e VAN DER POORTEN D, 2017; VANDENPLAS Y, et al., 2015).

A disbiose pode ser desenvolvida por diversos motivos, entre eles está o uso indiscriminado de antibióticos, anti-inflamatórios hormonais e não hormonais e laxantes; a idade avançada do paciente, pois o expõe por mais tempo a fatores externos; o pH intestinal; a disponibilidade de material fermentável; o estado imunológico do hospedeiro e a má digestão. Desse modo, cabe ressaltar que a disbiose provocará um quadro clínico marcado por gases, diarreia, constipação (DOS SANTOS MORAES M, et al., 2018).

É existente uma pandemia de obesidade atualmente. Sendo importante destacar que mais da metade da população obesa mundial desenvolve a síndrome metabólica, uma doença inflamatória crônica. Desse modo, o processo metabólico na microbiota intestinal é alterado e desequilibrado na vigência dessa síndrome por conta do estímulo à inflamação. Sendo assim, cabe considerar que a população bacteriana é alterada nos indivíduos obesos, provocando a disbiose, mas é possível que o próprio desequilíbrio da microbiota seja um fator-chave para desencadear a obesidade (CHANG C e LIN H, 2016; VIEIRA CR, et al., 2016).

Ademais, em casos extremos de obesidade, é indicada a cirurgia bariátrica que consiste na retirada parcial ou total do estômago, dependendo do método utilizado. A consequência é uma qualidade de vida melhor para o paciente e reeducação alimentar, melhorando, consequentemente, a disbiose (DA SILVA MARTINS N, et al., 2018).

Devido às influências de fatores ambientais na microbiota intestinal, diversos métodos são utilizados no tratamento da disbiose intestinal. As mudanças dietéticas podem afetar a composição bacteriana da microbiota intestinal, como o consumo de prebióticos e probióticos, ou a combinação de ambos (simbióticos). 
Além disso, o transplante da microbiota fecal vem sendo apresentado como uma nova abordagem terapêutica (SIDHU M e VAN DER POORTEN D, 2017; VANDENPLAS Y, et al., 2015; GALLEGO CG e SALMINEN S, 2016).

Assim, esse estudo objetivou revisar a literatura a fim de reunir informações atualizadas quanto ao diagnóstico e às condutas terapêuticas disponíveis na disbiose intestinal.

\section{MÉTODOS}

Foi realizada uma revisão sistemática da literatura, com intuito de capturar artigos que apresentassem em seus desenhos de estudo correlação com o tema. As informações foram obtidas a partir de buscas feitas nas bases de dados LILACS, MEDLINE e PUBMED, utilizando como descritores os termos "Disbiose", "Transplante de microbiótica fecal" e "Probióticos", com as opções em inglês, português, espanhol e francês, tendo sido empregado o boleano AND. Além disso, foi utilizado como critério de inclusão os artigos que possuíam boa confiabilidade e abordava e explanava o tema de forma adequada. Já os critérios de exclusão do trabalho foram os artigos publicados antes de 2014, artigos com amostras incompatíveis e artigos incompleto (Figura 1). Assim, foram encontrados 20 artigos aptos para serem abordados, sendo que 18 artigos foram publicados nos últimos 5 anos e apenas 2 artigos foram publicados nos últimos 6 anos.

Figura 1- Fluxograma das etapas da revisão sistemática.

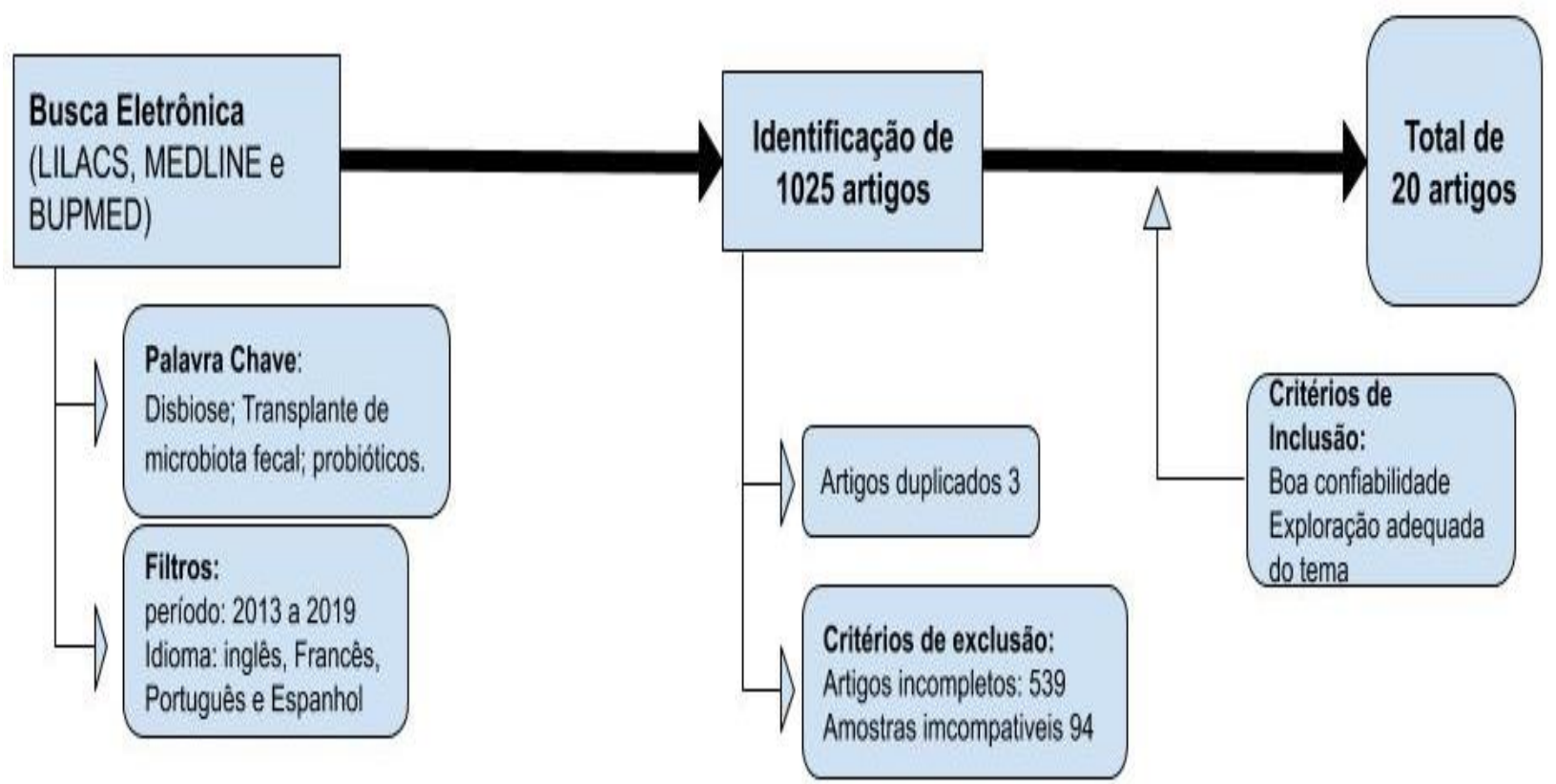

Fonte: Pantoja CL, Costa ACC, Costa PLS, et al., 2019.

\section{RESULTADOS}

Após aplicados os critérios de elegibilidade, 20 artigos foram identificados no período do estudo, publicados a partir de 2013, sendo 15 destes, publicados a partir de 2016, demonstrando que o tema ainda é atual. Ademais, dos artigos coletados, 5 relataram os sinais e sintomas presentes na disbiose intestinal, bem como fatores de grande importância clínica no quadro da doença.

São explanados artigos incluídos na revisão de acordo com autor, ano de publicação e tratamento abordado. Dessa forma, pode-se notar que 9 autores discutiram sobre o tratamento com probióticos, 5 discutiram sobre o tratamento por meio do transplante da microbiota fecal, 5 autores discutiram sobre 0 tratamento da disbiose com prebióticos, 3 abordaram sobre o tratamento com antibióticos e 3 explicaram sobre o tratamento por meio da suplementação de glutamina (Quadro 1). 
Quadro 1 - Artigos incluídos na revisão de acordo com autor, ano de publicação e tratamento abordado. Belém- PA, 2019.

\begin{tabular}{|c|c|c|c|c|c|}
\hline & Prebióticos & Probióticos & $\begin{array}{l}\text { Transplante da } \\
\text { microbiota fecal } \\
\text { (TMF) }\end{array}$ & $\begin{array}{l}\text { Suplementação } \\
\text { de Glutamina }\end{array}$ & $\begin{array}{l}\text { Tratamento com } \\
\text { antibiótico }\end{array}$ \\
\hline $\begin{array}{l}\text { AïT-AISSA A e } \\
\text { AIIDER M, } 2014 .\end{array}$ & - & SIM & - & - & - \\
\hline $\begin{array}{l}\text { BINDELS LB, et } \\
\text { al., 2016. }\end{array}$ & SIM & - & - & - & - \\
\hline $\begin{array}{c}\text { BRUNEAU A, et } \\
\text { al., } 2018 .\end{array}$ & - & - & SIM & - & - \\
\hline $\begin{array}{c}\text { CHOI HH e CHO } \\
\text { Y, } 2016\end{array}$ & - & - & SIM & - & SIM \\
\hline $\begin{array}{l}\text { COSTELLO SP, et } \\
\text { al., } 2015 .\end{array}$ & - & - & SIM & - & - \\
\hline $\begin{array}{l}\text { DE OLIVEIRA } \\
\text { GLV, et al., } 2017\end{array}$ & - & SIM & - & - & - \\
\hline $\begin{array}{l}\text { DOS SANTOS } \\
\text { MORAES M, et } \\
\text { al., } 2018 .\end{array}$ & - & SIM & - & SIM & - \\
\hline $\begin{array}{c}\text { GALLEGO CG e } \\
\text { SALMINEN S, } \\
2016 .\end{array}$ & SIM & SIM & - & - & - \\
\hline $\begin{array}{c}\text { HO KJ e VARGAS } \\
\text { J, } 2017 .\end{array}$ & - & - & - & - & SIM \\
\hline $\begin{array}{l}\text { HOLECEK M, } \\
2013 .\end{array}$ & - & - & - & SIM & - \\
\hline $\begin{array}{l}\text { MORROW LE e } \\
\text { WISCHMEYER P, } \\
2017 .\end{array}$ & - & SIM & - & - & - \\
\hline $\begin{array}{c}\text { PAIXÃO LA e } \\
\text { DOS SANTOS } \\
\text { CASTRO, FF, } \\
2016\end{array}$ & SIM & SIM & SIM & - & - \\
\hline $\begin{array}{c}\text { PEREIRA IG e } \\
\text { FERRAZ IAR, } \\
2017 .\end{array}$ & - & - & - & SIM & - \\
\hline $\begin{array}{l}\text { SAAD N, et al., } \\
2013 .\end{array}$ & SIM & SIM & - & - & - \\
\hline $\begin{array}{c}\text { SIDHU M e VAN } \\
\text { DER POORTEN } \\
\text { D, } 2017 .\end{array}$ & - & SIM & - & - & SIM \\
\hline $\begin{array}{l}\text { VANDENPLAS Y, } \\
\text { et al., } 2015 .\end{array}$ & - & SIM & SIM & - & - \\
\hline $\begin{array}{c}\text { VIEIRA CR, et al., } \\
2016 .\end{array}$ & SIM & - & - & - & - \\
\hline
\end{tabular}

Fonte: Pantoja CL, Costa ACC, Costa PLS, et al., 2019. 


\section{DISCUSSÃO}

A manifestação clínica da disbiose é representada, frequentemente, por diarréia, esteatorréia, distensão abdominal, gases, cólicas e constipação, sendo o diagnóstico estritamente clínico e correspondente ao quadro clínico da síndrome disabsortiva (LOPES CLR, et al., 2017).

Pode-se destacar um fator importante na contribuição para o desequilíbrio da microbiota intestinal, tal como a má absorção, pois o estômago produz ácido suficiente para extinguir as bactérias patogênicas ingeridas nos alimentos. Além disso, outros fatores que também têm importância clínica são o abuso do laxante, o consumo excessivo de alimentos crus, exposição com frequência a toxinas ambientais, disponibilidade de material fermentável e o estado imunológico do hospedeiro (PAIXÃO LA e DOS SANTOS CASTRO, FF, 2016)

Desse modo, deve haver uma investigação criteriosa a respeito do tipo de alimentação do indivíduo, serem destacados os fatores de risco e analisados criteriosamente todos os sinais e sintomas presentes para se realizar o diagnóstico. Sendo assim, é importante frisar que o mau hábito alimentar, a atividade física reduzida ou ausente, o uso constante e desregular de antibióticos e suplementos nutricionais podem corroborar com o desenvolvimento de doenças crônicas como alergias, obesidade e doença inflamatória intestinal. Essas doenças afetam diretamente o microbioma, provocando condições clínicas negativas para o paciente (PEREIRA IG e FERRAZ IAR, 2017; HOLECEK M, 2013).

Dessa forma, para o tratamento não ser agressivo nem invasivo, pode-se iniciá-lo por meio de manipulação dietética, incluindo a suplementação de Glutamina (GLN), um aminoácido, por auxiliar na funcionalidade das barreiras da mucosa intestinal e por ser fundamental para o bom desenvolvimento de tecido muscular intestinal. (PEREIRA IG e FERRAZ IAR, 2017; HOLECEK M, 2013).

\section{Tratamento com antibióticos}

O microbioma intestinal representa um ecossistema dinâmico que pode ser moldado por vários fatores, inclusive pelo uso de antibióticos (HO KJ e VARGAS J, 2017).

$\mathrm{Na}$ antibioticoterapia, é realizada a administração de metronidazol ou vancomicina e a remoção do antibiótico desencadeador das alterações no microbioma (SIDHU M e VAN DER POORTEN D, 2017).

Essas medidas são utilizadas no tratamento da infecção por Clostridium difficile, a qual está associada a disbiose intestinal, tendo como terapia padrão a vancomicina $500 \mathrm{mg}$ por via oral quatro vezes por dia durante 14 dias.

No entanto, tem se mostrado ineficiente nos casos de pacientes que possuem múltiplas recidivas, uma vez que a eficácia estimada do uso de vancomicina para uma primeira recidiva é de $60 \%$ ( $\mathrm{CHOI} \mathrm{HH} \mathrm{e} \mathrm{CHO}$ Y, 2016).

\section{Utilização de Probióticos}

Os probióticos são definidos como bactérias vivas ou leveduras que, quando administradas em quantidades adequadas, beneficiam a saúde do consumidor. Os probióticos bacterianos mais conhecidos são os Lactobacillus, Bifidobacterium e Lactococcus, enquanto a levedura mais utilizada é Saccharomyces boulardii (CHOI HH e CHO Y, 2016; DOS SANTOS MORAES M, et al., 2018; MORROW LE e WISCHMEYER $P, 2017)$.

Eles proporcionam efeitos benéficos na dor abdominal global, escores de inchaço e flatulência, mas podem visar além do trato gastrointestinal como: o trato respiratório, o trato urinário, a pele e a vagina, por exemplo, justamente por serem desenvolvidos para melhorar as doenças fisiológicas em diferentes áreas do corpo (MORROW LE e WISCHMEYER P, 2017).

Os mecanismos de ação dos probióticos contra patógenos consistem, principalmente, em: competição por nutrientes e sítios de acesso, produção de metabólitos antimicrobianos, mudanças nas condições ambientais e modulação da resposta imune do hospedeiro (PEREIRA IG e FERRAZ IAR, 2017). 
Essas ações ocorrem por meio do aumento da barreira intestinal natural, da estimulação da secreção de $\operatorname{lgA}$, da síntese de vitaminas, do aprimoramento do trânsito intestinal, o que aliviam intolerâncias à lactose, reduzem inchaço e promovem regulação negativa da produção de citocinas inflamatórias (SAAD N, et al., 2013; VANDENPLAS Y, et al., 2015).

Podem ser administrados em indivíduos saudáveis e doentes, tendo efeitos de natureza preventiva e curativa. No entanto, apesar de serem uma forma de tratamento segura para a maioria dos indivíduos, pode haver casos de infecção oportunista devido à interferência com a microflora comensal, particularmente em adultos criticamente doentes e neonatos (SAAD N, et al., 2013; VANDENPLAS Y, et al., 2015).

\section{Suplementação de Glutamina}

A glutamina (GLN) ajuda na potencialização dos efeitos dos probióticos no tratamento da disbiose intestinal. A GLN, aminoácido essencial e mais abundante no corpo humano, tem papel importante no metabolismo e transporte de nutrientes, funções imunes e integridade intestinal. Portanto, uma dieta enriquecida com GLN pode aumentar as atividades de transporte de borda em escova, além de melhorar o desempenho dos enterócitos (PEREIRA IG e FERRAZ IAR, 2017; HOLECEK M, 2013).

É possível destacar também a capacidade de auxiliar no processo de desenvolvimento e crescimento de massa muscular. Além disso, a suplementação de GLN reduz sintomas de doenças associadas à disbiose intestinal, como a síndrome do intestino irritável, doenças hepáticas e doença de Crohn (PEREIRA IG e FERRAZ IAR, 2017).

\section{Prebióticos}

Os prebióticos consistem em um ingrediente fermentado que possibilita mudanças na composição e na atividade da microbiota gastrointestinal, o que permite benefícios ao bem-estar e saúde do hospedeiro. A maioria desses benefícios está associada ao aumento da expressão ou à mudança na composição de ácidos graxos de cadeia curta, ao aumento do peso fecal, à redução do $\mathrm{pH}$ do cólon luminal, à diminuição nos produtos finais nitrogenados e enzimas redutoras e à modulação do sistema imunitário (BINDELS LB, et al., 2016; SAAD N, et al., 2013).

Os prebióticos no intestino tem se mostrado benéfico por promoverem o aumento de bifidobactérias e lactobacilos ou inibição de várias cepas de bactérias patogênicas humanas e animais, como Clostridium sp. e E. coli. Exemplo disso, é o fato da oligofrutose e / ou galacto-oligossacarídeos mostrarem ter um efeito sobre a recidiva da diarréia associada ao Clostridium difficile. Além disso, uma combinação de prebióticos, como polidextrose e lactitol tem aumentado a secreção de imunoglobulina A (IgA) (SAAD N, et al., 2013).

A maioria dos estudos sobre prebióticos tem se concentrado em frutanos, como os frutanos do tipo inulina, os galactooligossacáridos (FOS), os frutooligossacarídeos (FOS) e a lactulose. A aplicação dos prebióticos tem sido estudada, também, na prevenção de câncer, uma vez que as frações de fermentação do sobrenadante inulina apresentam efeitos inibidores de crescimento e indutivos de apoptose significativos no humano. Além disso, estudos recentes demonstraram interesse pelo efeito sob o metabolismo lipídico, adsorção mineral e efeito imuno-modulatórios dos prebióticos (AÏT-AISSA A e AÏDER M, 2014; GALLEGO CG e SALMINEN S, 2016; SAAD N, et al., 2013).

\section{Transplante da microbiota fecal (TMF)}

O TMF funciona por meio da infusão de fezes de um indivíduo doador no trato gastrointestinal de um paciente com, por exemplo, infecção por Clostridium difficile, a fim de restaurar a diversidade da microbiota intestinal. As fezes doadas são misturadas com solução salina / glicerol estéril e podem ser frescas ou congeladas, sendo que as fezes congeladas a $-80^{\circ} \mathrm{C}$ são viáveis e cultiváveis cerca de seis a dez meses. O transplante pode pela via sonda naso-jejunal ou no ceco na colonoscopia, sendo considerado um procedimento seguro. ocorrer Procedimento realizado, principalmente, em condições de difícil tratamento de situações nas quais se sabe que a microbiota fecal é anormal ( $\mathrm{CHOI} \mathrm{HH} \mathrm{e} \mathrm{CHO} \mathrm{Y,} \mathrm{2016;} \mathrm{COSTELLO} \mathrm{SP,} \mathrm{et}$ al., 2015; SIDHU M e VAN DER POORTEN D, 2017). 


\section{CONCLUSÃO}

A microbiota intestinal influencia diretamente na saúde do hospedeiro, sendo importante frisar que as bactérias podem ser patogênicas, mas também são essenciais à vida, devendo haver uma simbiose entre o hospedeiro e as bactérias, uma espécie de mutualismo, a qual ambos se beneficiem em prol da saúde do hospedeiro. Assim, a partir das informações reunidas da literatura, pode-se destacar que o diagnóstico da disbiose é puramente clínico e se faz necessária uma análise criteriosa dos sintomas e fatores desencadeadores da doença. Assim, entre as terapias utilizadas para essa doença, incluem uma alimentação rica em prebióticos e probióticos desde a infância e o uso de metronidazol e vancomicina de forma racional, equilibrando a microbiota do hospedeiro e prevenindo doenças intestinais de crianças, adultos e idosos. Ademais, o transplante da microbiota fecal também é uma forma terapêutica utilizada, mas é indicada em casos de difícil tratamento em infecção por Clostridium difficile ou em pacientes recidivantes.

\section{REFERÊNCIAS}

1. AÏT-AISSA A; AÏDER M. Lactulose: production and use in functional food, medical and pharmaceutical applications. Practical and critical review. International journal of food science \& technology, 2014; 49(5): 1245-1253.

2. BINDELS LB, et al. Towards a more comprehensive concept for prebiotics. Nature reviews Gastroenterology \& hepatology, 2016; 12(5): 303.

3. BRUNEAU $A$ et al. Le microbiote intestinal: quels impacts sur la carcinogenèse et le traitement du cancer colorectal?. Bulletin du Cancer, 2018; 105(1): 70-80.

4. CHANG C; LIN H. Dysbiosis in gastrointestinal disorders. Best Practice \& Research Clinical Gastroenterology, 2016; 30(1): 3-15.

5. $\mathrm{CHOI} \mathrm{HH;} \mathrm{CHO}$ Y. Fecal microbiota transplantation: current applications, effectiveness, and future perspectives. Clinical endoscopy, 2016; 49(3): 257.

6. COSTELLO SP, et al. Faecal microbiota transplant for recurrent Clostridium difficile infection using long-term frozen stool is effective: clinical efficacy and bacterial viability data. Alimentary pharmacology \& therapeutics, 2015; 42(8): 1011-1018.

7. DA SILVA MARTINS N, et al. Disbiose em pacientes bariátricos. RBONE-Revista Brasileira de Obesidade, Nutrição e Emagrecimento, 2018; 12(70): 145-154.

8. DE OLIVEIRA GLV, et al. Intestinal dysbiosis and probiotic applications in autoimmune diseases. Immunology, 2017; 152(1): 1-12.

9. DOS SANTOS MORAES M, et al. EFEITOS FUNCIONAIS DOS PROBIÓTICOS COM ÊNFASE NA ATUAÇÃO DO KEFIR NO TRATAMENTO DA DISBIOSE INTESTINAL. UNILUS Ensino e Pesquisa, 2018; 14(37); 144-156.

10. GALLEGO CG; SALMINEN S. Novel probiotics and prebiotics: how can they help in human gut microbiota dysbiosis?. Applied Food Biotechnology, 2016; 3(2): 72-81.

11. HO KJ; VARGA J. Early-Life Gut Dysbiosis: A Driver of Later-Life Fibrosis?. The Journal of investigative dermatology, 2017; 137(11): 2253-2255.

12. HOLECEK M. Side effects of long-term glutamine supplementation. Journal of parenteral and Enteral Nutrition, 2013; 37(5): 607-616.

13. LOPES CLR, et al. PREVALÊNCIA DE SINAIS E SINTOMAS DE DISBIOSE INTESTINAL EM PACIENTES DE UMA CLÍNICA EM TERSINA-PI. Ciência \& Desenvolvimento-Revista Eletrônica da FAINOR, 2017; 10(3).

14. MORROW LE; WISCHMEYER P. Blurred lines: dysbiosis and probiotics in the intensive care unit. Chest, 2017; 151(2): 492-499.

15. PAIXÃO LA; DOS SANTOS CASTRO, FF. Colonização da microbiota intestinal e sua influência na saúde do hospedeiro. Universitas: Ciências da Saúde, 2016; 14(1): 85-96.

16. PEREIRA IG; FERRAZ IAR. Suplementação de glutamina no tratamento de doenças associadas à disbiose intestinal. Revista Brasileira de Saúde Funcional, 2017; 1(1): 46.

17. SAAD N, et al. An overview of the last advances in probiotic and prebiotic field. LWT-Food Science and Technology, 2013; 50(1): 1-16.

18. SIDHU M; VAN DER POORTEN D. The gut microbiome. Aust Fam Physician, 2017; 46(4): 206-211.

19. VANDENPLAS Y, et al. Probiotics: an update. Jornal de Pediatria (Versão em português), 2015; 91(1): 6-21.

20. VIEIRA CR, et al. Efeito de uma bebida funcional à base de farinha de banana (Musa spp.) verde sobre a microbiota intestinal, níveis de citocinas e capacidade antioxidante em mulheres com excesso de peso e adiposidade abdominal. Dissertação (Mestrado em Biociências aplicadas à saúde) - Instituto de Ciências Biomédicas. Universidade Federal de Alfenas, Minas Gerais, 2016. 